\title{
On the $r$-Central Coefficient Matrices of the Catalan Triangles
}

\author{
Juan Yin and Sheng-Liang Yang \\ Department of Applied Mathematics, Lanzhou University of Technology, Lanzhou, Gansu 730050, China
}

Correspondence should be addressed to Juan Yin; yinjuan972815@163.com

Received 23 September 2014; Accepted 21 December 2014

Academic Editor: Gi Sang Cheon

Copyright (C) 2015 J. Yin and S.-L. Yang. This is an open access article distributed under the Creative Commons Attribution License, which permits unrestricted use, distribution, and reproduction in any medium, provided the original work is properly cited.

We introduce the definition of the $r$-central coefficient matrices of a given Riordan array. Applying this definition and Lagrange Inversion Formula, we can calculate the $r$-central coefficient matrices of Catalan triangles and obtain some interesting triangles and sequences.

\section{Introduction}

Riordan arrays have drawn the attention of various authors from many points of view in the recent literature. We recall some concepts and properties of Riordan arrays. An infinite lower triangular matrix $D=\left(d_{n, k}\right)_{n, k \geq 0}$ is called a Riordan array if the generating function of its column $k$ is $d(t) h(t)^{k}$, where $d(t)=\sum_{n=0}^{\infty} d_{n} t^{n}$ and $h(t)=\sum_{n=0}^{\infty} h_{n} t^{n}$ are formal power series with $d_{0}=1, h_{0}=0$, and $h_{1} \neq 0$. We also write $D=(d(t), h(t))$; the general term of matrix $D=\left(d_{n, k}\right)$ is $d_{n, k}=\left[t^{n}\right] d(t) h(t)^{k}$, where $\left[t^{n}\right]$ is the coefficient operator.

The set of all Riordan arrays forms a group under ordinary matrix multiplication. The group law is then given by

$$
(d(t), h(t))(g(t), f(t))=(d(t) g(h(t)), f(h(t))) .
$$

The identity is $(1, t)$ and the inverse of $(d(t), h(t))$ is

$$
(d(t), h(t))^{-1}=\left(\frac{1}{d(\bar{h}(t))}, \bar{h}(t)\right),
$$

where $\bar{h}(t)$ is compositional inverse of $h(t)$.

If we multiply the Riordan array $D=(d(t), h(t))$ by a column vector $\left(b_{0}, b_{1}, b_{2}, \ldots\right)^{T}$ with generating function $B(t)$, then we get a column vector whose generating function is given by $d(t) B(h(t))$. The rule can be rewritten as

$$
(d(t), h(t)) B(t)=d(t) B(h(t)),
$$

which is the Fundamental Theorem of the Riordan group.
Lemma 1 (see [1]). An infinite lower triangular matrix $D=$ $\left(d_{n, k}\right)_{n, k \geq 0}$ is a Riordan array if and only if there exists a sequence $A=\left(a_{0}, a_{1}, a_{2}, \ldots\right)$ such that

$$
\begin{array}{r}
d_{n+1, k+1}=a_{0} d_{n, k}+a_{1} d_{n, k+1}+a_{2} d_{n, k+2}+\cdots, \\
n, k=0,1,2, \ldots
\end{array}
$$

Such a sequence is called the A-sequence of the Riordan array $D$. Besides, the A-sequence is uniquely determined by the function $h(t)$ according to the following formula and vice versa:

$$
\begin{aligned}
& h(t)=t A(h(t)) \\
& \text { or } A(t)=\left[\frac{h(y)}{y} \mid t=h(y)\right]=\left[\frac{t}{y} \mid t=h(y)\right] .
\end{aligned}
$$

In the calculation below we need the Lagrange Inversion Formula and some knowledge of generalized binomial series.

Lemma 2 ((LIF) [2]). Supposing that a formal power series $w=w(t)$ is implicitly defined by the relation $w=t \phi(w)$, where $\phi(t)$ is a formal power series such that $\phi(0) \neq 0$; then for any formal power series $F(t)$,

$$
\varphi\left(\left[t^{n}\right] F(t)(\phi(t))^{n}\right)=\left[\frac{F(w)}{1-t \phi^{\prime}(w)} \mid w=t \phi(w)\right]
$$

where $\varphi\left(b_{n}\right)$ stands for the generating function for $\left(b_{n}\right)$; that is, $\varphi\left(b_{n}\right)=\sum_{n \geq 0} b_{n} t^{n}$. 
Lemma 3 (see $[2,3]$ ). For any integer $q$, the generalized binomial series $\mathscr{B}_{q}(t)$ is given by

$$
\mathscr{B}_{q}(t)=\sum_{n=0}^{\infty} \frac{1}{q n+1}\left(\begin{array}{c}
q n+1 \\
n
\end{array}\right) t^{n}
$$

for which we have

$$
\begin{aligned}
\left(\mathscr{B}_{q}(t)\right)^{x}= & \sum_{n=0}^{\infty} \frac{x}{q n+x}\left(\begin{array}{c}
q n+x \\
n
\end{array}\right) t^{n} \\
= & {\left[(1+w)^{x} \mid w=t(1+w)^{q}\right], } \\
& \mathscr{B}_{q}(t)=1+t \mathscr{B}_{q}(t)^{q} .
\end{aligned}
$$

Given a Riordan array $\left(d_{n, k}\right)_{n, k \geq 0}$, then its central coefficient matrix is defined as $\left(d_{2 n, n+k}\right)_{n, k \geq 0}$. For example, the central coefficient matrix of the Pascal matrix $P=\left(\left(\begin{array}{l}n \\ k\end{array}\right)\right)_{n, k \geq 0}$, which is one of the most classical matrices, plays an important role in combinatorics. The first few rows of its central coefficient matrix [4] are

$$
P^{[1]}=\left(\begin{array}{ccccc}
1 & 0 & 0 & 0 & 0 \\
2 & 1 & 0 & 0 & 0 \\
6 & 4 & 1 & 0 & 0 \\
20 & 15 & 6 & 1 & 0 \\
70 & 56 & 28 & 8 & 1
\end{array}\right)
$$

In [5], this matrix is used to give a new proof of an identity of Andrews [6]. In this paper, we describe a process of obtaining new Riordan matrices from a given Riordan array, which corresponds to taking the central coefficient matrix several times. By considering the Catalan triangles, we obtain some interesting triangles and sequences. The first Catalan triangle we consider is $A=\left(a_{n, k}\right)_{n, k \geq 0}$ with $a_{n, k}=((k+1) /(n+$ 1)) $\left(\begin{array}{c}2 n-k \\ n-k\end{array}\right)$, which was introduced by Aigner [7]. The first few rows are

$$
A=(C(t), t C(t))=\left(\begin{array}{cccccc}
1 & 0 & 0 & 0 & 0 & 0 \\
1 & 1 & 0 & 0 & 0 & 0 \\
2 & 2 & 1 & 0 & 0 & 0 \\
5 & 5 & 3 & 1 & 0 & 0 \\
14 & 14 & 9 & 4 & 1 & 0 \\
42 & 42 & 28 & 14 & 5 & 1
\end{array}\right)
$$

where $C(t)$ is the generation function of the Catalan numbers. The second Catalan triangle is $B=\left(b_{n, k}\right)_{n, k \geq 0}$ with $b_{n, k}=((k+$ $1) /(n+1))\left(\begin{array}{c}2(n+1) \\ n-k\end{array}\right), n, k \geq 0$, which was introduced by Shapiro [8]. The first few rows are

$$
B=\left(C(t)^{2}, t C(t)^{2}\right)=\left(\begin{array}{cccccc}
1 & 0 & 0 & 0 & 0 & 0 \\
2 & 1 & 0 & 0 & 0 & 0 \\
5 & 4 & 1 & 0 & 0 & 0 \\
14 & 14 & 6 & 1 & 0 & 0 \\
42 & 48 & 27 & 8 & 1 & 0 \\
132 & 165 & 110 & 44 & 10 & 1
\end{array}\right)
$$

The third Catalan triangle is $C=\left(c_{n, k}\right)_{n, k \geq 0}$ with $c_{n, k}=((2 k+$ 1)/(2n+1)) $\left(\begin{array}{c}2 n+1 \\ n-k\end{array}\right), n, k \geq 0$, which was introduced by Radoux [9]. The first few rows are

$$
C=\left(C(t), t C(t)^{2}\right)=\left(\begin{array}{cccccc}
1 & 0 & 0 & 0 & 0 & 0 \\
1 & 1 & 0 & 0 & 0 & 0 \\
2 & 3 & 1 & 0 & 0 & 0 \\
5 & 9 & 5 & 1 & 0 & 0 \\
14 & 28 & 20 & 7 & 1 & 0 \\
42 & 90 & 75 & 35 & 9 & 1
\end{array}\right)
$$

\section{The $r$-Central Coefficient Matrices of the Catalan Triangles}

Definition 4. Let $H=\left(h_{n, k}\right)_{n, k \geq 0}$ be a Riordan array; then we say that $H^{[r]}=\left(h_{2^{r} n,\left(2^{r}-1\right) n+k}\right)$ are the $r$-central coefficient matrices of $H$, where $n, k, r \in \mathbb{N}$ and $r \neq 0$.

According to the definition, the 0 -central coefficient matrix $H^{[0]}$ is $H$ itself; the 1 -central coefficient matrix $H^{[1]}$ is equal to central coefficient matrix. In genaral, the $(i+$ 1)-central coefficient matrix $H^{[i+1]}$ is the central coefficient matrix of $H^{[i]}$. For example, the first few rows of the 2-central coefficient matrix of Pascal matrix are

$$
P^{[2]}=\left(\begin{array}{ccccc}
1 & 0 & 0 & 0 & 0 \\
4 & 1 & 0 & 0 & 0 \\
28 & 8 & 1 & 0 & 0 \\
220 & 66 & 12 & 1 & 0 \\
1820 & 560 & 120 & 16 & 1
\end{array}\right)
$$

Theorem 5. The r-central coefficient matrices of $A=(C(t)$, $t C(t))$ can be written as

$$
\begin{aligned}
A^{[r]} & =\left(\tilde{a}_{n, k}\right)_{n, k \geq 0} \\
& =\left(\frac{\mathscr{B}_{2^{r}+1}(t)\left(2-\mathscr{B}_{2^{r}+1}(t)\right)}{2^{r}+1-2^{r} \mathscr{B}_{2^{r}+1}(t)}, t \mathscr{B}_{2^{r}+1}(t)^{2^{r}}\right) .
\end{aligned}
$$

Moreover the generating functions of the A-sequences of $A^{[r]}$ are $(1-t)^{-2^{r}}$.

Proof. On the one hand, for any $n, k, r \in \mathbb{N}, r \neq 0$, we have

$$
\begin{aligned}
\tilde{a}_{n, k} & =a_{2^{r} n,\left(2^{r}-1\right) n+k} \\
& =\frac{\left(2^{r}-1\right) n+k+1}{2^{r} n+1}\left(\begin{array}{c}
\left(2^{r}+1\right) n-k \\
n-k
\end{array}\right) \\
& =\left(1-\frac{n-k}{2^{r} n+1}\right)\left(\begin{array}{c}
2^{r} n+n-k \\
n-k
\end{array}\right) \\
& =\left(\begin{array}{c}
2^{r} n+n-k \\
n-k
\end{array}\right)-\left(\begin{array}{c}
2^{r} n+n-k \\
n-k-1
\end{array}\right) \\
& =\left[t^{n-k}\right](1+t)^{2^{r} n+n-k}-\left[t^{n-k}\right] t(1+t)^{2^{r} n+n-k}
\end{aligned}
$$




$$
\begin{aligned}
& =\left[t^{n-k}\right](1+t)^{\left(2^{r}+1\right) n-k}(1-t) \\
& =\left[t^{n}\right](1+t)^{\left(2^{r}+1\right) n}\left(\frac{t}{1+t}\right)^{k}(1-t) \\
& \stackrel{(\mathrm{LIF})}{=}\left[t^{n}\right]\left[\frac{(1-w)(w /(1+w))^{k}}{1-t\left(2^{r}+1\right)(1+w)^{2^{r}}} \mid w=t(1+w)^{2^{r}+1}\right] .
\end{aligned}
$$

By (9) and (10), we have

$$
\begin{aligned}
\widetilde{a}_{n, k} & =\left[t^{n}\right] \frac{\mathscr{B}_{2^{r}+1}(t)\left(2-\mathscr{B}_{2^{r}+1}(t)\right)}{2^{r}+1-2^{r} \mathscr{B}_{2^{r}+1}(t)}\left(\frac{\mathscr{B}_{2^{r}+1}(t)-1}{\mathscr{B}_{2^{r}+1}(t)}\right)^{k} \\
& =\left[t^{n}\right] \frac{\mathscr{B}_{2^{r}+1}(t)\left(2-\mathscr{B}_{2^{r}+1}(t)\right)}{2^{r}+1-2^{r} \mathscr{B}_{2^{r}+1}(t)}\left(t \mathscr{B}_{2^{r}+1}(t)^{2^{r}}\right)^{k} .
\end{aligned}
$$

On the other hand, let $h(t)=t \mathscr{B}_{2^{r}+1}(t)^{2^{r}}=\left(\mathscr{B}_{2^{r}+1}(t)-\right.$ 1) $/ \mathscr{B}_{2^{r+1}}(t)=1-1 / \mathscr{B}_{2^{r}+1}(t)$, which implies $h(t)=t(1 /(1-$ $h(t)))^{2^{r}}$; by (5), we finish the proof.

Theorem 6. The inverse Riordan arrays of $A^{[r]}$ can be written as

$$
\left(A^{[r]}\right)^{-1}=\left(\frac{\left(1-2^{r} t-t\right)(1-t)}{1-2 t}, t(1-t)^{2^{r}}\right)
$$

Proof. Let $A^{[r]}=(d(t), h(t))=\left(\mathscr{B}_{2^{r}+1}(t)\left(2-\mathscr{B}_{2^{r}+1}(t)\right) /\left(2^{r}+\right.\right.$ $\left.\left.1-2^{r} \mathscr{B}_{2^{r}+1}(t)\right), t \mathscr{B}_{2^{r}+1}(t)^{2^{r}}\right)$. Then $\bar{h}(t)=t(1-t)^{2^{r}}$. We have known that $\mathscr{B}_{2^{r}+1}(t)=1 /(1-h(t))$; then $d(t)=((1 /(1-$ $h(t)))((1-2 h(t)) /(1-h(t)))) /\left(\left(1-2^{r} h(t)-h(t)\right) /(1-h(t))\right)=$ $(1-2 h(t)) /\left(1-2^{r} h(t)-h(t)\right)(1-h(t))$ and $1 / d(\bar{h}(t))=(1-$ $\left.2^{r} t-t\right)(1-t) /(1-2 t)$. Hence the result follows by (2).

The first few rows of $A^{[1]}$ and $A^{[2]}$ are

$$
\begin{aligned}
A^{[1]} & =\left(\begin{array}{ccccc}
1 & 0 & 0 & 0 & 0 \\
2 & 1 & 0 & 0 & 0 \\
9 & 4 & 1 & 0 & 0 \\
48 & 20 & 6 & 1 & 0 \\
275 & 110 & 35 & 8 & 1
\end{array}\right) ; \\
A^{[2]} & =\left(\begin{array}{ccccc}
1 & 0 & 0 & 0 & 0 \\
4 & 1 & 0 & 0 & 0 \\
35 & 8 & 1 & 0 & 0 \\
350 & 77 & 12 & 1 & 0 \\
3705 & 798 & 135 & 16 & 1
\end{array}\right) .
\end{aligned}
$$

Theorem 7. The r-central coefficient matrices of $B=\left(C(t)^{2}\right.$, $\left.t C(t)^{2}\right)$ can be written as

$$
\begin{aligned}
B^{[r]} & =\left(\widetilde{b}_{n, k}\right)_{n, k \geq 0} \\
& =\left(\frac{\mathscr{B}_{2^{r+1}}(t)^{2}\left(2-\mathscr{B}_{2^{r+1}}(t)\right)}{2^{r+1}-\left(2^{r+1}-1\right) \mathscr{B}_{2^{r+1}}(t)}, t \mathscr{B}_{2^{r+1}}(t)^{r^{r+1}}\right),
\end{aligned}
$$

and the generation functions of the A-sequences of $B^{[r]}$ are $(1+$ $t)^{2^{r+1}}$.
Proof. On the one hand, for any $n, k, r \in \mathbb{N}, r \neq 0$, we have

$$
\begin{aligned}
\widetilde{b}_{n, k} & =b_{2^{r} n,\left(2^{r}-1\right) n+k} \\
& =\frac{2^{r} n+1-n+k}{2^{r} n+1}\left(\begin{array}{c}
2\left(2^{r} n+1\right) \\
n-k
\end{array}\right) \\
& =\left(1-\frac{n-k}{2^{r} n+1}\right)\left(\begin{array}{c}
2^{r+1} n+2 \\
n-k
\end{array}\right) \\
& =\left[t^{n}\right](1+t)^{2^{r+1} n}(1+t) t^{k}(1-t) \\
& \stackrel{\operatorname{LIF}}{=}\left[t^{n}\right]\left[\frac{(1+w)(1-w) w^{k}}{1-2^{r+1} t(1+w)^{2^{r+1}-1}} \mid w=t(1+w)^{2^{r+1}}\right] \\
& =\left[t^{n}\right] \frac{\mathscr{B}_{2^{r+1}}(t)^{2}\left(2-\mathscr{B}_{2^{r+1}}(t)\right)}{2^{r+1}-\left(2^{r+1}-1\right) \mathscr{B}_{2^{r+1}}(t)}\left(t\left(\mathscr{B}_{2^{r+1}}(t)\right)^{2^{r+1}}\right)^{k} .
\end{aligned}
$$

On the other hand, let $f(t)=t\left(\mathscr{B}_{2^{r+1}}(t)\right)^{2^{r+1}}=\mathscr{B}_{2^{r+1}}(t)-$ 1, which implies $f(t)=t(1+f(t))^{2^{r+1}}$; by (5) we finish the proof.

Theorem 8. The inverse Riordan arrays of $B^{[r]}$ can be written as

$$
\left(B^{[r]}\right)^{-1}=\left(\frac{\left(1-\left(2^{r+1}-1\right) t\right)}{(1+t)^{2}(1-t)}, \frac{t}{(1+t)^{2^{r+1}}}\right) .
$$

Proof. Let $B^{[r]}=(g(t), f(t))=\left(\mathscr{B}_{2^{r+1}}(t)^{2}\left(2-\mathscr{B}_{2^{r+1}}(t)\right) /\right.$ $\left.\left(2^{r+1}-\left(2^{r+1}-1\right) \mathscr{B}_{2^{r+1}}(t)\right), t \mathscr{B}_{2^{r+1}}(t)^{2^{r+1}}\right)$. Then $\bar{f}(t)=t /(1+$ $t)^{2^{r+1}}$. Since $\mathscr{B}_{2^{r+1}}(t)=f(t)+1$, then $g(t)=\left(1+f(t)^{2}(1-\right.$ $f(t))) /\left(1-\left(2^{r+1}-1\right) f(t)\right)$ and $1 / g(\bar{f}(t))=\left(1-\left(2^{r+1}-1\right) t\right) /(1+$ $t)^{2}(1-t)$. Hence the result follows by (2).

The first few rows of $B^{[1]}$ and $B^{[2]}$ are

$$
\begin{aligned}
B^{[1]} & =\left(\begin{array}{ccccc}
1 & 0 & 0 & 0 & 0 \\
4 & 1 & 0 & 0 & 0 \\
27 & 8 & 1 & 0 & 0 \\
208 & 65 & 12 & 1 & 0 \\
1700 & 544 & 119 & 16 & 1
\end{array}\right) ; \\
B^{[2]} & =\left(\begin{array}{ccccc}
1 & 0 & 0 & 0 & 0 \\
8 & 1 & 0 & 0 & 0 \\
119 & 16 & 1 & 0 & 0 \\
2000 & 275 & 24 & 1 & 0 \\
35464 & 4928 & 495 & 32 & 1
\end{array}\right) .
\end{aligned}
$$

Theorem 9. The $r$-central coefficient matrices of $C=(C(t)$, $\left.t C(t)^{2}\right)$ can be written as

$$
\begin{aligned}
C^{[r]} & =\left(\widetilde{c}_{n, k}\right)_{n, k \geq 0} \\
& =\left(\frac{\mathscr{B}_{2^{r+1}}(t)\left(2-\mathscr{B}_{2^{r+1}}(t)\right)}{2^{r+1}-\left(2^{r+1}-1\right) \mathscr{B}_{2^{r+1}}(t)}, t \mathscr{B}_{2^{r+1}}(t)^{2^{r+1}}\right),
\end{aligned}
$$

and the generation functions of the A-sequences of $C^{[r]}$ are $(1+$ $t)^{2^{r+1}}$. 
Proof. For any $n, k, r \in \mathbb{N}, r \neq 0$, we have

$$
\begin{aligned}
& \widetilde{c}_{n, k}=c_{2^{r} n,\left(2^{r}-1\right) n+k} \\
& =\frac{2^{r+1} n-2 n+2 k+1}{2^{r+1} n+1}\left(\begin{array}{c}
2^{r+1} n+1 \\
n-k
\end{array}\right) \\
& =\left(1-\frac{2(n-k)}{2^{r+1} n+1}\right)\left(\begin{array}{c}
2^{r+1} n+1 \\
n-k
\end{array}\right) \\
& =\left(\begin{array}{c}
2^{r+1} n+1 \\
n-k
\end{array}\right)-2\left(\begin{array}{c}
2^{r+1} n \\
n-k-1
\end{array}\right) \\
& =\left[t^{n}\right](1+t)^{2^{r+1} n} t^{k}(1-t) \\
& \stackrel{\operatorname{LIF}}{=}\left[t^{n}\right]\left[\frac{(1-w) w^{k}}{1-2^{r+1} t(1+w)^{2^{r+1}-1}} \mid w=t(1+w)^{2^{r+1}}\right] \\
& =\left[t^{n}\right] \frac{\mathscr{B}_{2^{r+1}}(t)\left(2-\mathscr{B}_{2^{r+1}}(t)\right)}{2^{r+1}-\left(2^{r+1}-1\right) \mathscr{B}_{2^{r+1}}(t)}\left(t\left(\mathscr{B}_{2^{r+1}}(t)\right)^{2^{r+1}}\right)^{k} \text {. }
\end{aligned}
$$

Hence we finish the proof.

Theorem 10. The inverse Riordan arrays of $C^{[r]}$ can be written as

$$
\left(C^{[r]}\right)^{-1}=\left(\frac{\left(1-\left(2^{r+1}-1\right) t\right)}{(1+t)(1-t)}, \frac{t}{(1+t)^{2^{r+1}}}\right) .
$$

Proof. This can be easily obtained from the proof of Theorem 8.

The first few rows of $C^{[1]}$ and $C^{[2]}$ are

$$
\begin{aligned}
C^{[1]} & =\left(\begin{array}{ccccc}
1 & 0 & 0 & 0 & 0 \\
3 & 1 & 0 & 0 & 0 \\
20 & 7 & 1 & 0 & 0 \\
154 & 54 & 11 & 1 & 0 \\
1260 & 440 & 104 & 15 & 1
\end{array}\right) ; \\
C^{[2]} & =\left(\begin{array}{ccccc}
1 & 0 & 0 & 0 & 0 \\
7 & 1 & 0 & 0 & 0 \\
104 & 15 & 1 & 0 & 0 \\
1748 & 252 & 23 & 1 & 0 \\
31000 & 4464 & 464 & 31 & 1
\end{array}\right)
\end{aligned}
$$

\section{Conflict of Interests}

The authors declare that there is no conflict of interests regarding the publication of this paper.

\section{Acknowledgments}

The authors would like to thank the referees for their helpful suggestions. This work was supported by the National Natural Science Foundation of China (Grant no. 11261032).

\section{References}

[1] T.-X. He and R. Sprugnoli, "Sequence characterization of Riordan arrays," Discrete Mathematics, vol. 309, no. 12, pp. 39623974, 2009.

[2] D. Merlini, R. Sprugnoli, and M. C. Verri, "Lagrange inversion: when and how," Acta Applicandae Mathematicae, vol. 94, no. 3, pp. 233-249, 2006.

[3] G.-S. Cheon and L. Shapiro, "The uplift principle for ordered trees," Applied Mathematics Letters, vol. 25, no. 6, pp. 1010-1015, 2012.

[4] A. Luzon, D. Merlini, M. Moron, and R. Sprugnoli, "Identities induced by Riordan arrays," Linear Algebra and Its Applications, vol. 436, no. 3, pp. 631-647, 2012.

[5] E. H. Brietzke, "An identity of Andrews and a new method for the Riordan array proof of combinatorial identities," Discrete Mathematics, vol. 308, no. 18, pp. 4246-4262, 2008.

[6] G. E. Andrews, "Some formulae for the Fibonacci sequence with generalizations," The Fibonacci Quarterly, vol. 7, no. 2, pp. 113$130,1969$.

[7] M. Aigner, "Enumeration via ballot numbers," Discrete Mathematics, vol. 308, no. 12, pp. 2544-2563, 2008.

[8] L. W. Shapiro, "A Catalan triangle," Discrete Mathematics, vol. 14, no. 1, pp. 83-90, 1976.

[9] C. Radoux, "Additional formulas for polynomials built on classical combinatorial eqquences," Journal of Computational and Applied Mathematics, vol. 115, no. 1-2, pp. 471-477, 2000. 


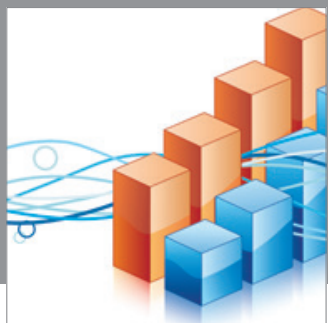

Advances in

Operations Research

mansans

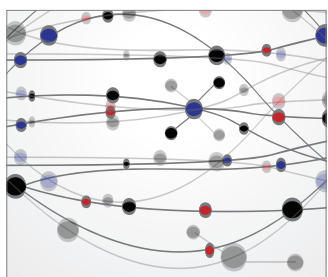

The Scientific World Journal
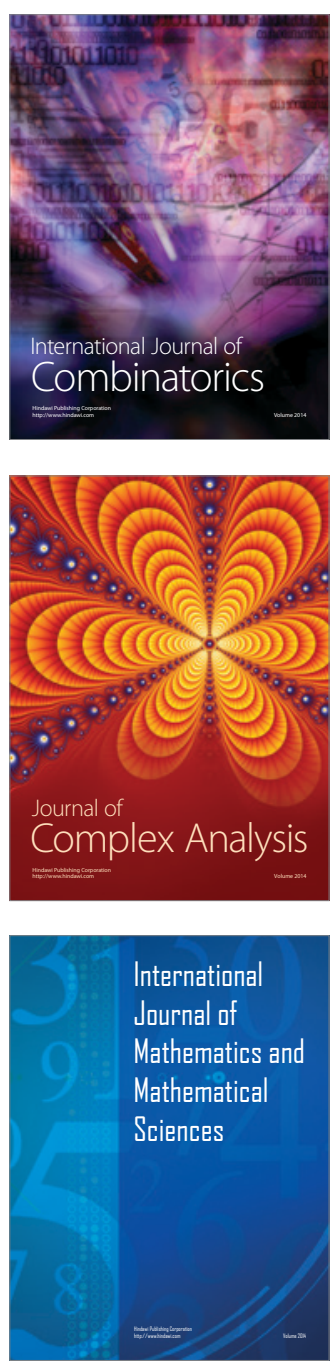
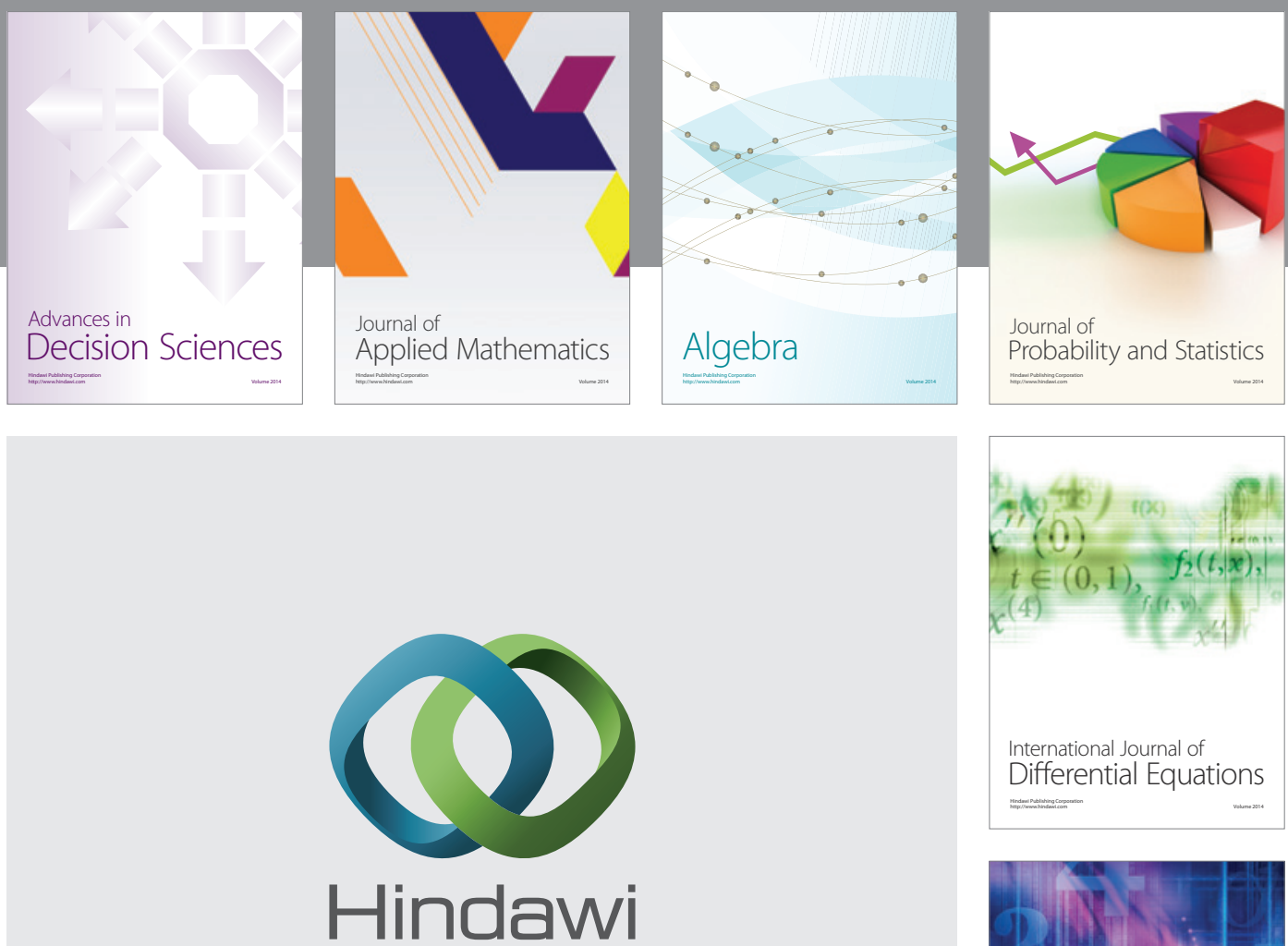

Submit your manuscripts at http://www.hindawi.com
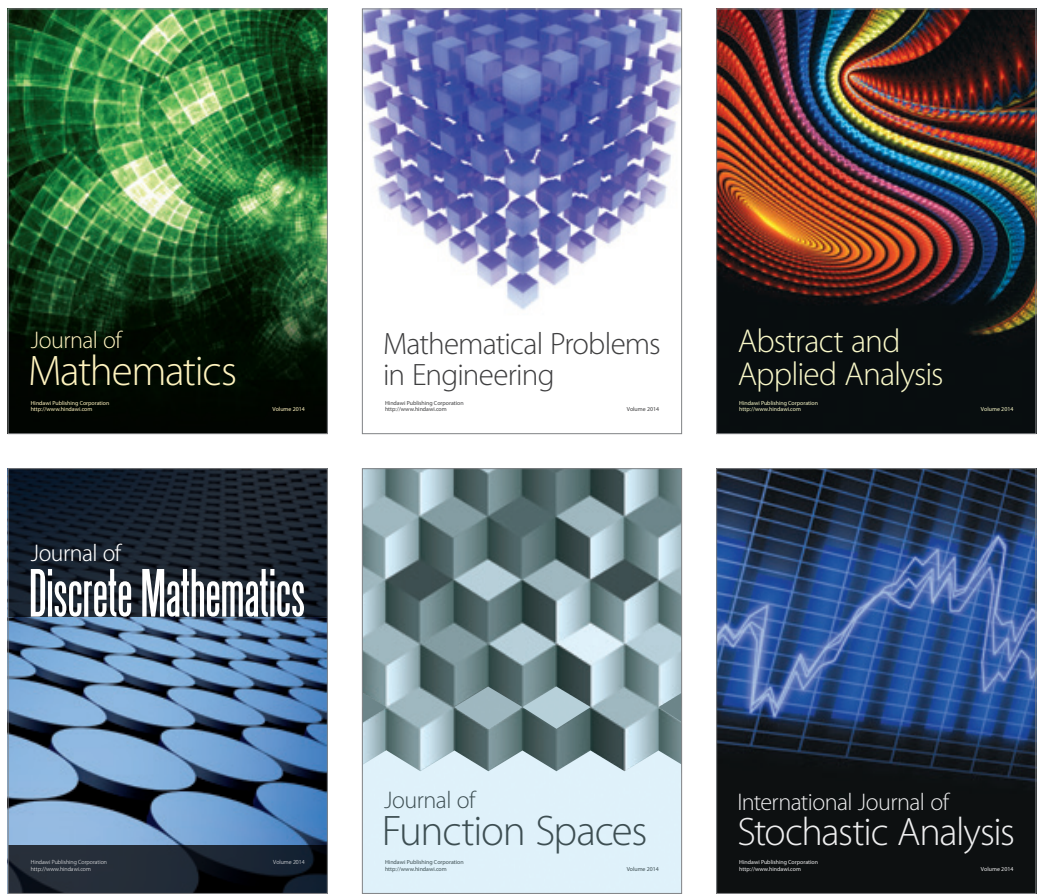

Journal of

Function Spaces

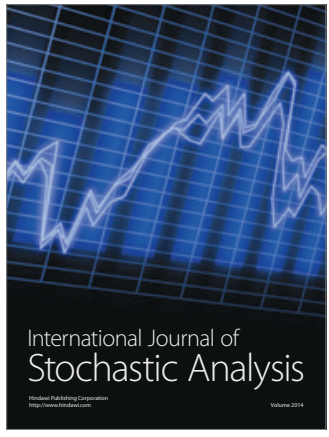

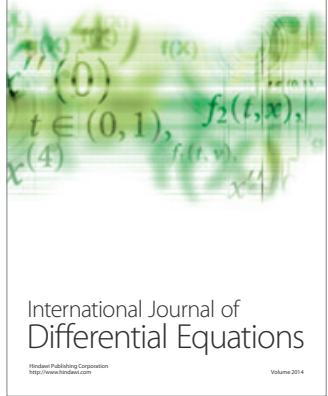
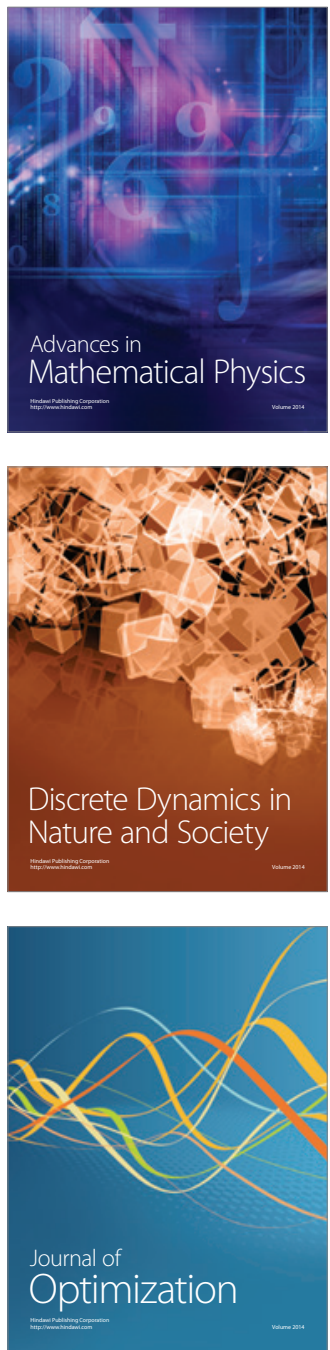